\title{
The deltopectoral flap in the management of tracheostomal stenosis post laryngectomy and radiotherapy
}

\author{
D Liakos, ${ }^{1,2}$ (D) C Sofianos, ${ }^{2}$ (D) DRW Dower, ${ }^{1}$ (i) P Ciudad, ${ }^{1}$ (i) HC Chen ${ }^{1,3}$ (iD \\ ${ }^{1}$ Department of Reconstructive Microsurgery, China Medical University Hospital, Taiwan \\ ${ }^{2}$ Department of Plastic and Reconstructive Surgery, University of the Witwatersrand, South Africa \\ ${ }^{3}$ International Medical Service Center, China Medical University Hospital, Taiwan
}

Corresponding author, email: sofianosc@gmail.com

Background: Tracheostomal stenosis is a distressing complication with a high incidence rate post laryngectomy. We aimed to assess the deltopectoral flap (DPF) for tracheostomal stenosis correction in irradiated patients.

Methods: Six patients with tracheostomal stenosis, 3 of whom developed restenosis after prior use of local flaps, were managed using a DPF to reconstruct the defect following a vertical incision release of the stenotic band down to the inferior tracheal cartilaginous ring. Healing absence of restenosis, alleviation of the symptoms of difficulty in breathing and clearance of secretions were considered a successful composite endpoint.

Results: Over a median follow-up period of 11 months all patients maintained patency, and symptoms of difficulty in breathing and clearing of secretions were alleviated.

Conclusion: The deltopectoral flap is a rapid, reliable flap for the management of tracheostomal stenosis in irradiated patients. It brings well-vascularised tissue into the site of reconstruction and, in the short term, stomal patency and symptom relief were achieved.

Keywords: deltopectoral, DP, flap, tracheostomal, stenosis, radiation

\section{Introduction}

Tracheostomal stenosis (TS) is a distressing complication in patients with permanent tracheostomies. Patients may have trouble clearing secretions and may present with respiratory distress. ${ }^{1,2}$ The incidence of TS has been reported to be as high as $55 \%$ with a median value of $28.4 \%$, depending on patient profile and associated risk factors. ${ }^{1}$ Many factors have been implicated in TS, including postoperative infection, keloid formation, steroid use, site of stomal placement, primary trachea-oesophageal puncture, postoperative fistula formation, extensive neck dissection and radiation therapy. ${ }^{1,3}$ Management of TS includes stenting, dilatation, surgical correction or a combination of the above. Possibly the most important factor in prevention of tracheal stenosis is the surgical technique used in the tracheostomy. Circular transection of the trachea should be avoided, and a plastic closure is reported to have the lowest incidence of stenosis. ${ }^{4-6}$

Many techniques have been published for the management of TS. These mainly include a variety of local tissue flaps, including variations of Z-plasty techniques, V-shaped flap and the petal technique.,.$^{2,47-9}$ The deltopectoral flap (DPF) is a well-described flap and has been shown to be reliable in head and neck reconstruction. Furthermore, it is an axially-based flap as opposed to the random-pattern flaps listed above. The DPF has been described for primary tracheostomal reconstruction, ${ }^{10,11}$ but not specifically for the management of TS.
We present the outcomes of using the DPF for the management of TS in patients who had undergone laryngectomy and radiation therapy.

\section{Materials and methods}

We report on six patients who were managed for TS with the use of a pedicled DPF for the period January 1999 July 2015. All the patients in the study group were male, five patients were diagnosed with hypopharyngeal squamous cell carcinoma and one patient with laryngeal squamous cell carcinoma. All patients received neo-adjuvant radiation therapy. Five patients were treated with total laryngopharyngectomies and bilateral neck dissection, and one patient was treated with total laryngectomy. Following permanent tracheostomies, patients presented with tracheostomal stenosis which caused difficulty breathing and clearing secretions. In addition, one patient required stenting for longer than three months to maintain adequate respiration. Time to revision is noted in Table 1.

In this study, reconstruction of the hypopharynx was either with a free ileocolon flap or free jejunal flap. Three of the six patients previously underwent attempts at TS release and reconstruction with the use of local flaps (Table 1).

Otolaryngology surgeons performed all the permanent tracheostomies and patients were eating well on a solid diet at the time of DPF reconstruction. Patients were followedup at the outpatient department at 1 week, 3 weeks, and then monthly postoperatively. 
Table 1: Patient demographics and reconstructive history

\begin{tabular}{cclll}
\hline Patient & Age & Reconstruction of primary tumour & Previous TS procedure & Time to stenosis* \\
\hline 1 & 49 & Free jejunal flap & None & 18 months \\
2 & 52 & Free ileocolon flap & Release + local flap & 13 months $\dagger$ \\
3 & 49 & Free ileocolon flap & None & 6 months \\
4 & 60 & Free ileocolon flap & Release + local flap & $<6$ months $\dagger$ \\
5 & 45 & Free ileocolon flap & Release + local flap & $<6$ months $\dagger$ \\
6 & 60 & Free ileocolon flap & None & 12 months
\end{tabular}

*Time to TS severe enough to cause clinical symptoms from the time of tracheostomy

†Time to stenosis from local flap reconstruction

\section{Technique}

Operations on patients were carried out under general anaesthesia. The patient is placed supine for both elevation and inset of the flap. The flap can be raised from either side of the chest. The TS was released in all patients using a vertical incision, releasing the stenotic band and the inferior tracheal cartilaginous ring. Adequate release of the stenosis was assessed clinically, taking into account the pre- and postoperative diameters of the stoma. The DPF was then marked out, taking into account the length of the flap required to transpose into the created defect and the width of the flap necessary for primary closure, taking into account the quality and excess of the surrounding skin. The flap was not designed longer than a point distal to the deltopectoral groove, as this would make the skin paddle unreliable distally. The flap was raised, and the perforators visualised and preserved.

Sutures were placed at the edges of the tracheostomy before the inset of the flap; this is a very important step to ensure adequate suturing of the flap to the tracheostomal edges. Polydioxanone sutures (PDS) were used. Elevation of the DPF is through standard techniques, starting laterally and proceeding medially, along a subfascial plane. Figure 1.4 shows the flap inset onto the tracheostomal edges that were identified previously. Once the flap is inset, the donor site was closed primarily or with a split-thickness skin graft. Patients were stented postoperatively for 2 to 3 weeks to prevent any airway compromise in the event of any complications.

\section{Results}

The median age of the patient group was 50 years (range 45-60 years). The median follow-up period was 11 months (range 5-24 months). There were no flap or donor site complications and all wounds healed well. Three donor sites were closed primarily, and three patients required a small splitthickness skin graft.

Upon follow-up, patients had no further difficulty breathing or clearing secretions and maintained wide stomas. One patient complained of some bulkiness of the flap in the stoma and this patient underwent a debulking procedure under local anaesthesia.

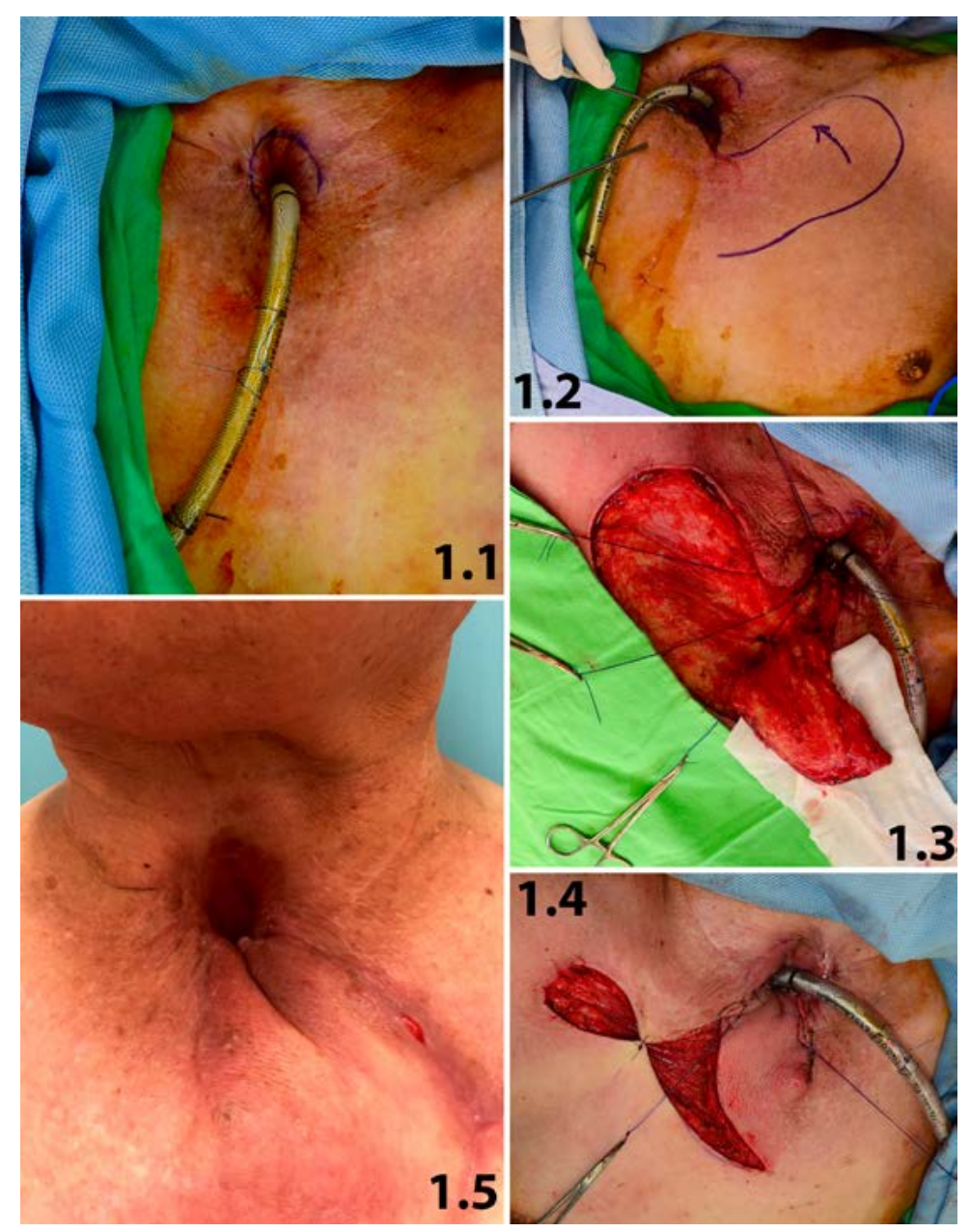

Figure 1: Case example of patient number 4 (Table 1).

Fig 1.1: Preoperative image of patient with TS. The constriction can be seen with an endotracheal tube in situ. Patient had previous attempt at stomaplasty with local flap reconstruction.

Fig 1.2: The vertical incision releasing the TS and the cartilage ring of the trachea and the design of the DPF.

Fig 1.3: Placement of sutures at the edges of the tracheostomy with the DPF raised.

\section{Fig 1.4: The DPF inset and the donor site closed primarily.}

Fig 1.5: The patient can be seen 1 month postoperatively, the patency of the stoma was maintained and the symptoms were relieved. 
All five patients experienced relief from distress whilst breathing and did not require postoperative stenting for longer than 3 weeks; all were satisfied with the outcome. Assessment of satisfaction was based on subjective symptom relief, as reported by the patients, and review for stridor or breathing difficulty by our surgical team. Two patients required a voice prosthesis for speaking, namely the patient who had a free jejunal flap for hypopharyngeal reconstruction and the patient with a total laryngectomy.

\section{Discussion}

The DPF is a well-described and reliable flap in head and neck reconstruction. ${ }^{12-14}$ McCarthy et al. reported its usefulness and applicability for flap coverage and tracheostomal reconstruction in this type of patient. ${ }^{12}$ Although initial reports of high complications were described by East in using the DPF for tracheostomal reconstruction, ${ }^{11}$ this was due to fenestration of the flap, which compromised its vascularity. Modifications of this flap have renewed applications in head and neck reconstruction. ${ }^{13}$

However, to our knowledge, the use of the DP flap and its outcomes in patients with tracheostomal stenosis specifically has not been described. Our research provides a novel approach for the management of patients with TS, and although our sample number is small, it has been shown to successfully manage the difficult scenario of TS in patients who have received radiation.

It is a reliable flap, which can be harvested easily and rapidly. It is thin and pliable and can be inset comfortably once the stomal stenosis is released. Another advantage of the DPF is that it brings well-vascularised tissue to the site of reconstruction in patients who have received radiation therapy.

Three of our patients had previous attempts at stomaplasty with stenosis release and local flap reconstruction; however, within six months these patients required revision with a DPF due to restenosis. All patients maintained adequate patency of the tracheostomy after DPF reconstruction during the follow-up period. The mean follow-up period of 11 months is shorter than desired as restenosis can occur up to 2 years postoperatively. Typical or expected timing to restenosis after secondary TS repair has not been previously documented. Madariaga et al. give average expected time to restenosis after primary tracheal repair and indicated that early stenosis occurs by two weeks, while late stenosis usually occurs by three months. ${ }^{15}$ Tissue injury due to radiotherapy is ongoing and chronic, thus a longer followup is required and will be the basis of future reports as the cohort expands and matures.

An objective method for assessment of stoma patency, would be a measurement of stomal size, with a stoma of less than $10 \mathrm{~mm} \times 10 \mathrm{~mm}$ regarded as narrow. ${ }^{16}$ The lack of objective, quantifiable methods of stomal patency assessment are a limitation of our current study and would be taken into account with longer follow-up in future assessments. A validated scoring system that includes both subjective symptom relief and objective stoma patency would have utility in follow-up.

Wax et al. described the management of TS with the use of dilatation and local flap reconstruction. ${ }^{4}$ They reported a success rate of more than $90 \%$ with local flap reconstruction; however, no mention was made of whether patients received radiation therapy. The concerns with the use of local flaps in tracheostomal reconstruction in patients undergoing radiation therapy are the damage and fibrosis to the surrounding tissues. ${ }^{17}$ This inevitably results in radiation damage to the local flaps that are used in stomaplasty. Furthermore, Giacomarra reported encouraging results with the use of local flap reconstruction in 12 patients $;{ }^{2}$ however, these patients had no preoperative radiation therapy.

Although Wax initially reported that radiation therapy does not play a role in stenosis of the tracheostomy, ${ }^{1}$ Griffith et al.showed that any radiation therapy causes concentric stenosis. ${ }^{3}$ Other risk factors for TS in this group of patients include diabetes mellitus and local infection as described by De Virgilio et al. ${ }^{18}$ In our small study group, all six patients developed restenosis after local flap reconstruction. Depending on the width of the flap which is raised, some folding of the flap can occur during inset. However, this can be easily debulked under local anaesthesia at a later stage.

Yet another advantage of the DPF is that it can be readvanced without compromise of vascularity if required. Furthermore, the pectoralis major musculocutaneous flap is preserved and is available, if necessary, for further head and neck reconstruction.

A small case series of two patients has been reported with the use of the internal mammary artery perforator (IMAP) flap in tracheostomal reconstruction, citing as an advantage primary closure of the donor site. ${ }^{19}$ The IMAP flap is a perforator based on the same blood supply as the DPF. It is based on either the second or third internal mammary artery perforators and is an islanded flap, in contrast to the DPF.

Local flaps should be considered the first line in the management of tracheostomal stenosis; however, as indicated earlier, in cases of recurrent stenosis and/or radiation to the area, local flaps are not desirable; the DPF offers a source of vascularised tissue that has not been subjected to radiation injury. Another flap that could be used in the management of these complex patients is the transverse cervical artery flap; this requires further study. Use of simpler techniques such as dilation, ${ }^{20}$ or carbon dioxide laser stomaplasty ${ }^{21}$ have little utility in recurrent TS in radiotherapy fields.

In three of our patients, the donor site was closed primarily without any wound complications, furthermore slight tension on closure added additional traction to the stoma, which increased the aperture. Depending on the width of the flap required for reconstruction and the surrounding skin laxity, a skin graft may be required for closure of the donor site. In the three patients requiring skin grafting, the donor sites healed well and posed no aesthetic concern to the patients. Skin grafts may be serially excised in the future, which may further improve the aesthetic appearance.

Because the DPF maintains a skin bridge as opposed to the IMAP flap, there is less risk of pedicle kinking or twisting and increased venous drainage in the DPF. There is no need to dissect out the perforators, which may prolong the procedure, as is necessary in the IMAP flap. Further, there is no need for preoperative Doppler examination when raising the DPF and it can be raised rapidly even under local anaesthesia.

Voice reconstruction should also be taken into account in patients undergoing total laryngectomies. Patients were able to use a voice prosthesis adequately after stenosis release and DP flap reconstruction. Our primary choice for hypopharyngeal reconstruction, however, is an ileocolon flap, as the colon is used for the pharyngeal reconstruction 
and the ileum is used for voice reconstruction after appropriate voice training. ${ }^{22}$

\section{Conclusion}

Our results suggest the DPF is a reliable flap for the management ofTS, especially in patients who have undergone radiation therapy. Patients maintain stomal patency after prolonged follow-up and do not require stenting. The DPF has the advantage of bringing well-vascularised tissue to the site of reconstruction in patients who have undergone radiation therapy and has now become our first choice in treating patients with tracheostomal stenosis.

\section{Conflict of interest}

The authors have no conflict of internet to declare.

\section{Funding source}

None.

\section{Ethics approval}

This work has ethical approval from the Institutional Review Board at China Medical University Hospital, Taiwan.

\section{ORCID}

D Liakos (iD) https://orcid.org/0000-0001-7612-6345

C Sofianos (D) https://orcid.org/0000-0002-3438-508X

DRW Dower (ID https://orcid.org/0000-0002-5586-1735

P Ciudad (D) https://orcid.org/0000-0003-4646-2676

HC Chen (iD https://orcid.org/0000-0002-2749-1909

\section{REFERENCES}

1. Wax MK, Touma BJ, Ramadan HH. Tracheostomal stenosis after laryngectomy: incidence and predisposing factors. Otolaryngol Head Neck Surg. 1995;113(3):242-7. doi:10.1016/S0194-5998(95)70112-5. PMID:7675484.

2. Giacomarra V, Russolo M, Tirelli G, Bonini P. Surgical treatment of tracheostomal stenosis. Laryngoscope. 2001;111(7):1281-4. doi:10.1097/00005537-20010700000026. PMID:11568555.

3. Griffith GR, Luce EA. Tracheal stomal stenosis after laryngectomy. Plast Reconstr Surg. 1982;70(6):694-8. PMID:7146151.

4. Wax MK, Touma BJ, Ramadan HH. Management of tracheostomal stenosis. Laryngoscope. 1999;109(9):1397-401. doi:10.1097/00005537-199909000-00006. PMID:10499042.

5. Hartwell SW, Jr., Dykes ER. Construction and care of the end tracheostomy. Am J Surg. 1967;113(4):498-500. PMID:6023023.

6. Suzuki M, Tsunoda A, Shirakura S, et al. A novel permanent tracheostomy technique for prevention of stomal stenosis (triangular tracheostomy). Auris Nasus Larynx. 2010;37(4):465-8. doi:10.1016/j.anl.2009.11.007. PMID:20036475.

7. Kim YH, Kim NH, Seong SY, Hyun DW, Choi HS. Double reversing Z-plasty with inferiorly widening stomaplasty for the management of tracheostomal stenosis. Auris Nasus
Larynx. 2010;37(3):361-4. doi:10.1016/j.anl.2009.10.002 PMID:20042304.

8. Lucioni M, Rizzotto G, Pazzaia T, Serafini I. Plastic tracheostomal-widening procedure: the "petal" technique. Acta Otorhinolaryngol Ital. 2003;23(4):291-6. PMID:15046418.

9. Vlantis AC, Marres HA, Van den Hoogen FJ. A surgical technique to prevent tracheostomal stenosis after laryngectomy. Laryngoscope. 1998;108(1 Pt 1):134-7. PMID:9432083.

10. Pay AD, Marucci DD, Morritt DG. Combined pectoralis major myocutaneous and fenestrated deltopectoral flaps for salvage tracheostomal, pharyngeal and neck skin reconstruction. J Plast Reconstr Aesthet Surg. 2009;62(1):127-8. doi:10.1016/j. bjps.2007.12.075. PMID:18848816.

11. East CA, Flemming AF, Brough MD. Tracheostomal reconstruction using a fenestrated deltopectoral skin flap. J Laryngol Otol. 1988;102(3):282-3. PMID:3356943.

12. McCarthy CM, Kraus DH, Cordeiro PG. Tracheostomal and cervical esophageal reconstruction with combined deltopectoral flap and microvascular free jejunal transfer after central neck exenteration. Plast Reconstr Surg. 2005;115(5):1304-10; discussion 11-3. PMID:15809590.

13. Kingdom TT, Singer MI. Enhanced reliability and renewed applications of the deltopectoral flap in head and neck reconstruction. Laryngoscope. 1996;106(10):1230-3. PMID: 8849791.

14. Tiwari RM, Gorter H, Snow GB. Experiences with the deltopectoral flap in reconstructive surgery of the head and neck. Head Neck Surg. 1981;3(5):379-83. PMID:7239936.

15. Madariaga ML, Gaissert HA. Reresection for recurrent stenosis after primary tracheal repair. J Thorac Dis. 2016;8(Suppl 2):S153-9. doi:10.3978/j.issn.2072-1439.2016.01.66. PMID: 26981266.

16. Bajaj Y, Shayah A, Loke D, et al. Long-term results with a simple technique of stoma creation after laryngectomy. Eur Arch Otorhinolaryngol. 2009;266(6):879-82. doi:10.1007/ s00405-008-0805-7 PMID: 18807063.

17. Bernstein EF, Sullivan FJ, Mitchell JB, Salomon GD, Glatstein E. Biology of chronic radiation effect on tissues and wound healing. Clin Plast Surg. 1993;20(3):435-53. PMID:8324983.

18. De Virgilio A, Greco A, Gallo A, et al. Tracheostomal stenosis clinical risk factors in patients who have undergone total laryngectomy and adjuvant radiotherapy. Eur Arch Otorhinolaryngol. 2013;270(12):3187-9. doi:10.1007/ s00405-013-2695-6. PMID:24057098.

19. Yu P, Roblin P, Chevray P. Internal mammary artery perforator (IMAP) flap for tracheostoma reconstruction. Head Neck. 2006;28(8):723-9. doi:10.1002/hed.20386. PMID:16619279.

20. Wu TH, Lee SC. A simple method for dilating tracheostomal stenosis. Clin Otolaryngol. 2010;35(4):342. doi:10.1111/ j.1749-4486.2010.02158.x. PMID:20738352.

21. Sani A. Carbon dioxide laser stomaplasty for tracheostomal stenosis. J Laryngol Otol. 1998;112(5):467-8. doi:10.1017/ s0022215100140794. PMID:9747477.

22. Karri V, Yang MC, Chung KP, et al. Total pharyngolaryngectomy and voice reconstruction with ileocolon free flap: functional outcome and quality of life. J Plast Reconstr Aesthet Surg. 2011;64(7):911-20. doi:10.1016/j.bjps.2010.11.019. PMID:21236743. 